\title{
Sea level measurements using multi-frequency GPS and GLONASS observations
}

\author{
Johan S Löfgren * and Rüdiger Haas
}

\begin{abstract}
Global Positioning System (GPS) tide gauges have been realized in different configurations, e.g., with one zenith-looking antenna, using the multipath interference pattern for signal-to-noise ratio (SNR) analysis, or with one zenith- and one nadir-looking antenna, analyzing the difference in phase delay, to estimate the sea level height. In this study, for the first time, we use a true Global Navigation Satellite System (GNSS) tide gauge, installed at the Onsala Space Observatory. This GNSS tide gauge is recording both GPS and Globalnaya Navigatsionnaya Sputnikovaya Sistema (GLONASS) signals and makes it possible to use both the one- and two-antenna analysis approach. Both the SNR analysis and the phase delay analysis were evaluated using dual-frequency GPS and GLONASS signals, i.e., frequencies in the L-band, during a 1-month-long campaign. The GNSS-derived sea level results were compared to independent sea level observations from a co-located pressure tide gauge and show a high correlation for both systems and frequency bands, with correlation coefficients of 0.86 to 0.97 . The phase delay results show a better agreement with the tide gauge sea level than the SNR results, with root-mean-square differences of $3.5 \mathrm{~cm}\left(G P S L_{1}\right.$ and $\left.L_{2}\right)$ and $3.3 / 3.2 \mathrm{~cm}$ (GLONASS $L_{1} / L_{2}$ bands) compared to 4.0/9.0 cm (GPS $\left.L_{1} / L_{2}\right)$ and $4.7 / 8.9 \mathrm{~cm}\left(G L O N A S S ~ L_{1} / L_{2}\right.$ bands). GPS and GLONASS show similar performance in the comparison, and the results prove that for the phase delay analysis, it is possible to use both frequencies, whereas for the SNR analysis, the $L_{2}$ band should be avoided if other signals are available. Note that standard geodetic receivers using code-based tracking, i.e., tracking the un-encrypted $C / A$-code on $L_{1}$ and using the manufacturers' proprietary tracking method for $L_{2}$, were used. Signals with the new $C / A$-code on $L_{2}$, the so-called $L_{2}$, were not tracked. Using wind speed as an indicator for sea surface roughness, we find that the SNR analysis performs better in rough sea surface conditions than the phase delay analysis. The SNR analysis is possible even during the highest wind speed observed during this campaign $(17.5 \mathrm{~m} / \mathrm{s})$, while the phase delay analysis becomes difficult for wind speeds above $6 \mathrm{~m} / \mathrm{s}$.
\end{abstract}

Keywords: Sea level; GNSS; GNSS-R; GPS; GLONASS; Signal-to-noise ratio; Geodetic analysis; Phase delay; Multi-frequency; Tide gauge

\section{Introduction}

The technique of observing sea level and its changes with the freely available Global Navigation Satellite System (GNSS) signals was first suggested by [1]. Since then, several studies of ocean remote sensing with Global Positioning System (GPS) signals involving land-based, airborne, and spaceborne systems have been carried out, e.g., [2,3], and [4], respectively. The advantage of using reflected GNSS signals for remote sensing of the sea surface, compared to measurements by traditional tide gauges, is the

*Correspondence: johan.lofgren@chalmers.se

Chalmers University of Technology, Department of Earth and Space Sciences, Onsala Space Observatory, Onsala SE-439 92, Sweden possibility of measuring absolute sea level, i.e., sea level with respect to the International Terrestrial Reference Frame (ITRF). Sea level measurements from traditional tide gauges are relative to the land where they are established, and they are therefore affected by land surface changes. However, for applications related to changes in the global ocean volume, e.g., the global sea level budget, and for sea level measurements in tectonically active regions, absolute sea level measurements are necessary [5]. Since GNSS can be used to measure land surface changes, e.g., [6], one possibility to achieve absolute sea level is to combine tide gauge measurements of sea level with GNSS measurements of land motion (see e.g., $[7,8]$ ). The assumption made in this case is that the GNSS station

\section{是 Springer}

(c) 2014 Löfgren and Haas; licensee Springer. This is an Open Access article distributed under the terms of the Creative Commons Attribution License (http://creativecommons.org/licenses/by/2.0), which permits unrestricted use, distribution, and reproduction in any medium, provided the original work is properly credited. 
is close enough to the tide gauge to be affected by the same land motion. Another possibility, involving only one technique, is to use GNSS signals for both measuring the sea level and the land motion, i.e., to use a so-called GNSS tide gauge.

GNSS tide gauges have been realized in different configurations, e.g., using one or two antennas and with different types of receivers. The one-antenna configuration builds upon using the signal interference pattern, originating from the otherwise unwanted multipath signals interfering with the direct GNSS signals (see, e.g., $[9,10]$ ), and was proposed by [11-13]. In later studies, GPS stations with zenith-looking antennas, installed primarily for geodetic measurements, have been used to measure the sea level of the nearby ocean (see [14-16]). For these types of studies, the interference pattern of the signal-to-noise ratio (SNR) is usually recorded with a geodetic receiver and analyzed with an interferometric approach. In addition, other studies exist, where the SNR of the direct and reflected signals was recorded by a customized receiver connected to an antenna directed towards the horizon (see e.g., [17]).

In the two-antenna configuration, one antenna is right-handed circularly polarized (RHCP) and directed upwards, receiving the direct satellite signals, and one antenna is left-handed circularly polarized (LHCP) and directed downwards, receiving the satellite signals that are reflected off the sea surface. GNSS carrier-phase measurements are recorded either by customized receivers and analyzed using interferometric techniques, e.g., [18,19], or by geodetic receivers and analyzed with a standard geodetic approach [20-22].

At the Onsala Space Observatory (OSO) in Sweden, a GNSS tide gauge installation was established dedicated to measuring reflected GNSS signals from the ocean. This installation supports both the one- and two-antenna approach and records both GPS and Globalnaya Navigatsionnaya Sputnikovaya Sistema (GLONASS) signals, making it a true GNSS tide gauge.

In the present study, we use the GNSS tide gauge at OSO in order to evaluate both the SNR analysis technique (one antenna) and the geodetic analysis technique (two antennas) and to investigate their performances and restrictions. For this evaluation, we utilize the full potential of the GNSS tide gauge, i.e., recording and analyzing signals from multiple systems with multiple frequencies. For the first time, in addition to using reflected GPS signals, also reflected GLONASS signals are used for measuring the local sea level. Furthermore, for both systems, signals from the frequency bands $L_{1}$ and $L_{2}$ are recorded and analyzed, and the results are compared with independent measurements from a co-located pressure sensor gauge.

Section 2 gives a description of the GNSS tide gauge at OSO together with details on the GNSS and the pressure tide gauge dataset used for this study. The two techniques and the respective data analysis are described in Sections 3 and 4, with the sea level results presented in Section 5. This is followed by a discussion in Section 6 and, finally, the conclusions and outlook in Section 7.

\section{The GNSS tide gauge at the Onsala Space Observatory}

The current GNSS tide gauge was installed at OSO on the west coast of Sweden $\left(57.4^{\circ} \mathrm{N}, 11.9^{\circ} \mathrm{E}\right)$ in the fall of 2011. It consists of two geodetic-type antennas mounted back-to-back on a beam extending over the coastline (see Figure 1 for a panoramic view of the installation site). The top antenna is RHCP and zenith-looking, and the bottom antenna is LHCP and nadir-looking. Both antennas are of the model Leica AR25 multi-GNSS choke ring (Leica Geosystems, Heerbrugg, St. Gallen, Switzerland), and both are covered by hemispherical radomes. Each antenna is connected to a Leica GRX1200GGPRO GNSS receiver recording data with $1-\mathrm{Hz}$ sampling rate. For this study, we used GPS and GLONASS carrier-phase and SNR data (resolution $0.25 \mathrm{~dB}-\mathrm{Hz}$ ) in both L-band frequency bands, known as $\mathrm{L}_{1}$ and $\mathrm{L}_{2}$, that were collected during 1 month from September 29, day-of-year (doy) 273 , to October, doy 303, 2012. The receivers used codebased tracking to track and record the carrier phase and SNR data, i.e., tracking the un-encrypted C/A-code on $\mathrm{L}_{1}$ and using the manufacturers' proprietary tracking method for $\mathrm{L}_{2}$. Signals with the new $\mathrm{C} / \mathrm{A}$-code on $\mathrm{L}_{2}$, the so-called $\mathrm{L}_{2 C}$, were not tracked. The reasons were that we wanted to maximize the number of GPS observations (there are only a few GPS satellites with $\mathrm{L}_{2 C}$ capability so far) and to have a consistent $\mathrm{L}_{2}$ dataset.

The GNSS tide gauge installation is directed towards the open water surface to the south to maximize the number of reflected satellite observations (see Figure 1). To the east, the water surface is limited by bedrock, and to the west, the water surface is limited by a beach and a few smaller islands. Nonetheless, the open water surface has a radius of more than $100 \mathrm{~m}$ and extends from azimuth angles $60^{\circ}$ to $300^{\circ}$. The mean vertical position of the upward-looking antenna during the campaign was approximately $4.3 \mathrm{~m}$ above the sea surface.

To the east of the GNSS tide gauge installation (approximately $10 \mathrm{~m}$ ), three hydrostatic level transmitters (HLT), i.e., pressure sensors, are installed. The sensors are mounted together on a submerged pole and measure the pressure from the overlying column of water (see e.g., [23]). The sensor type is Mobrey series 9710 HLT (Emerson Process Management Mobrey Ltd., Slough, Berkshire, $\mathrm{UK})$, and the temporal resolution is set to 1 sample per second. The output value consist of the mean over $60 \mathrm{~s}$, so the actual temporal resolution is 1 sample per minute. 


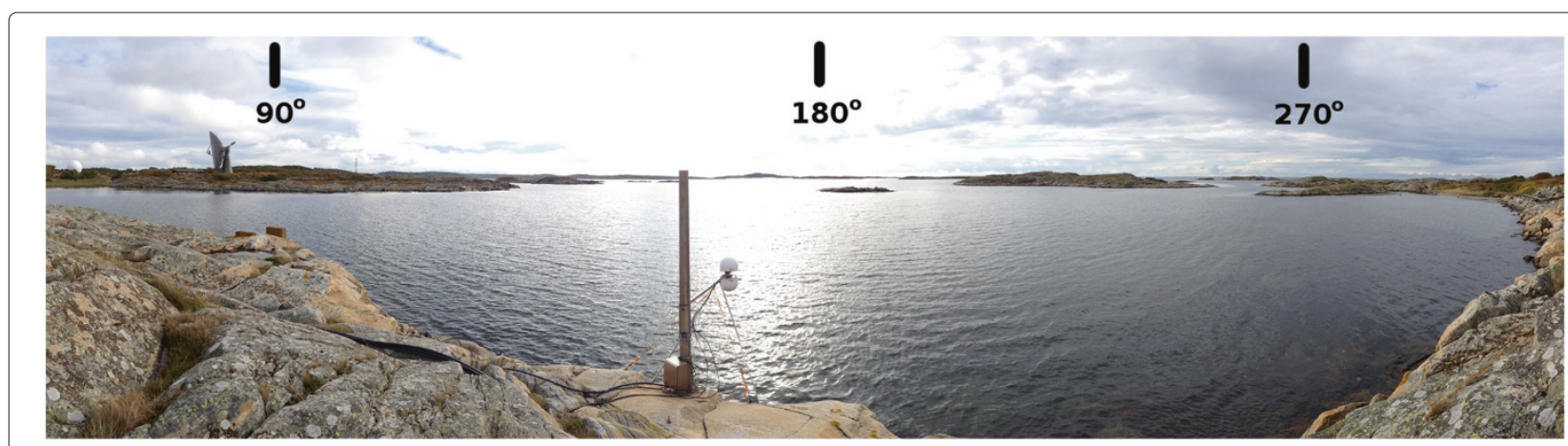

Figure 1 Panoramic view of the GNSS tide gauge at the Onsala Space Observatory. The installation is directed towards the south to receive the reflected GNSS signals from the open water surface (middle). Approximate azimuth directions are indicated in the top of the picture, showing that the open sea extends from azimuth $60^{\circ}$ to $300^{\circ}$. To the east (left) and west (right), the water surface is confined by bedrock.

\section{Analysis methods}

As previously described, the concept of the GNSS tide gauge can be realized in different ways. In this study, two techniques were investigated: 1) the SNR analysis using one zenith-looking antenna connected to a geodetic GNSS receiver and 2) the geodetic phase delay analysis using both a zenith-looking and a nadir-looking antenna, each connected to a geodetic GNSS receiver (see Figure 2). In both analysis techniques, the satellite signals reflected off the sea surface are used to estimate the sea level. However, the techniques take advantage of two different satellite observations, i.e., the SNR and the phase delay data, recorded by one or both of the receivers.

Both the SNR and the phase delay analysis have been described before, e.g., the SNR analysis in $[14,16]$ and the phase delay analysis in [22,24]. However, for the sake of completeness, both analysis methods are summarized below.

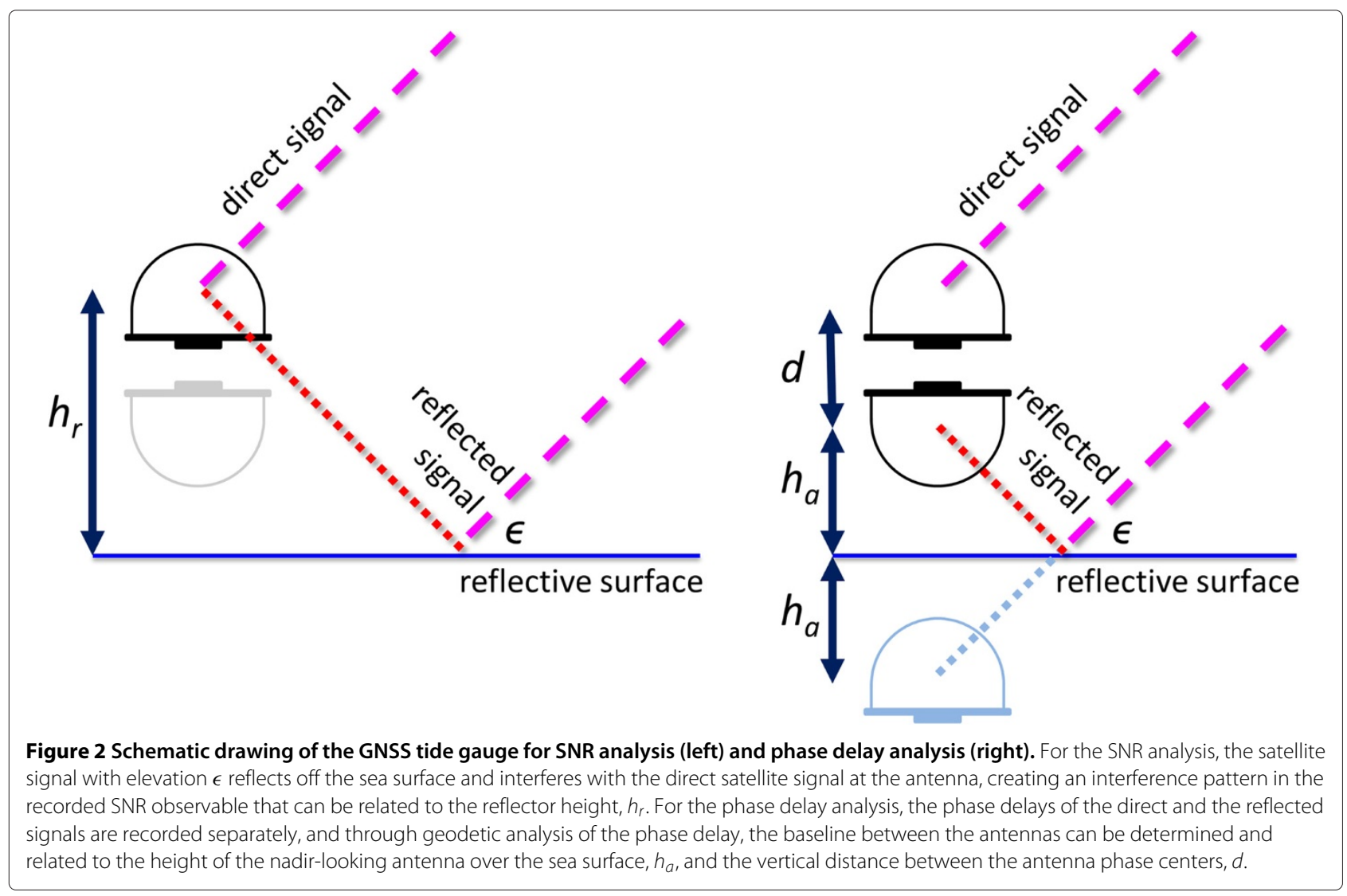




\subsection{The SNR analysis method}

GNSS antennas are by design sensitive to the direct satellite signals, suppressing the unwanted signals that are reflected in the surrounding environment before reaching the antenna. Nevertheless, some of the reflected signals interfere with the direct signals which affect the GNSS observables recorded by the receiver (see Figure 2 (left) for an illustration of a zenith-pointing antenna). This effect, known as multipath, is one of the major error sources in high-accuracy positioning, and there are several studies on how to mitigate the effect, e.g., [9,10,25]. In addition, there are studies on how to use the reflected signals to measure properties of the reflecting surface, e.g., sea level $[13,14,16]$, soil moisture [26], and snow depth [27].

When a GNSS satellite moves across the sky, the phase difference in the receiver between the direct and the reflected satellite signals changes, creating an interference pattern. This pattern is especially visible in the SNR data recorded by the receiver, and as an example, the SNR data from two satellite observations affected by multipath are presented in Figure 3 (left). Note that the SNR data in Figure 3 (left) are smoothed with a 30-s moving average filter (the original SNR data have a resolution of $0.25 \mathrm{~dB}$ $\mathrm{Hz}$ and a sampling rate of $1 \mathrm{~s}$ ) to improve the visibility. This is, however, not necessary for the SNR analysis.

There are two main characteristics of the SNR data in Figure 3 (left): the SNR multipath oscillations and the overall trend. The multipath effect is decreasing with increasing satellite elevation angle, i.e., the amplitude of the interference pattern decreases with increasing satellite elevation angle. This decrease depends on the amplitude (signal strength) of the reflected signal and the antenna gain pattern. For low satellite elevation angles, the reflected signal is mostly RHCP due to the higher amplitude of the RHCP Fresnel reflection coefficient compared to the LHCP coefficient (see e.g., $[22,25])$. However, the RHCP reflection coefficient decreases with increasing elevation angle.

The overall trend of the SNR arc (see Figure 3, left) depends on the receiver-satellite distance, the atmospheric attenuation, and the receiving antenna gain pattern. In order to isolate the multipath contribution to the SNR observation, the overall trend can be removed by either fitting and removing a low-order polynomial (see e.g., [28]) or by filtering the SNR signal (e.g., [29]). The remaining detrended SNR $(\delta \mathrm{SNR})$, which consists of the multipath oscillations, can be described by

$$
\delta \mathrm{SNR}=A \cos \left(\frac{4 \pi h_{r}}{\lambda_{i}} \sin \epsilon+\varphi\right)
$$

where $A$ is the amplitude, $h_{r}$ is the distance between the reflecting surface and the antenna phase center (also called reflector height; see Figure 2, left), $\lambda_{i}$ is the carrier wavelength of the GNSS signal, $\epsilon$ is the satellite elevation angle, and $\varphi$ is a phase offset.

Assuming that the reflector height (e.g., the sea level) is not changing during the satellite arc and that the reflector (e.g., the sea surface) is horizontal, the frequency of the
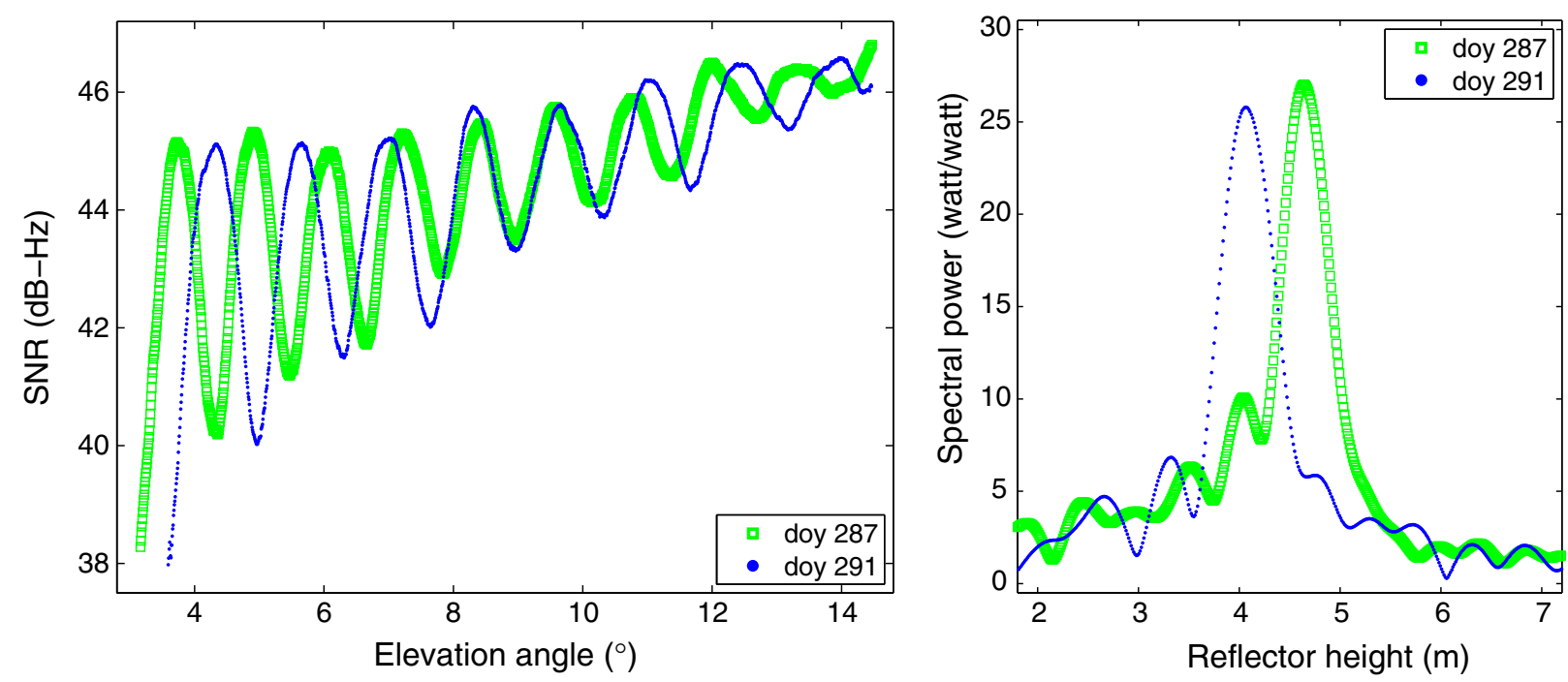

Figure 3 SNR measurements affected by multipath (left) and results from LSP analysis of the same data (right). The SNR measurements are recorded by the receiver connected to the zenith-looking antenna of the GNSS tide gauge. The signals are from the same satellite but for two different times, day-of-year (doy) 287 and 291, 2012. At doy 287, the sea level was lower (or the reflector height was larger) than for doy 291. This can be interpreted from the higher frequency of the multipath oscillations for doy 287, as compared to doy 291 . The SNR data were then detrended and analyzed with an LSP showing clear peaks for reflector heights of 4.64 and $4.07 \mathrm{~m}$ for doy 287 and 291, respectively. 
multipath oscillations $\left(2 h_{r} / \lambda_{i}\right)$ is constant with respect to the sine of the satellite elevation angle. It is also possible to model a reflector height that is changing during a satellite arc [15]. However, this is not necessary for stations with a sub-diurnal tidal range of less than about $2.7 \mathrm{~m}$ [16].

By spectral analysis of the $\delta$ SNR data as a function of sine of elevation angle, it is possible to derive the dominant multipath frequency. This is usually performed using the Lomb-Scargle periodogram (LSP), since it can handle unevenly spaced samples (the SNR data are evenly sampled in time, but not as a function of sine of elevation). As an example, Figure 3 (right) depicts the LSP results after analysis of the SNR data in Figure 3 (left) (note that the SNR data were first detrended). The peaks of the LSP in Figure 3 (right) correspond to the dominant multipath frequencies and thus the reflector heights. The reflector height is negatively correlated with sea level, e.g., a large reflector height, or high multipath frequency, corresponds to a large distance between the antenna and the sea surface, which means a low sea level.

\subsection{The phase delay analysis method}

To achieve high-accuracy positioning with GNSS, analysis of phase delay data is necessary. For the GNSS tide gauge, the setup consists of one zenith-looking antenna, recording the direct satellite signals which are RHCP, and one nadir-looking antenna, recording the satellite signals that are reflected off the sea surface which are mostly LHCP (see Figure 2 (right) for an illustration). As previously described, the RHCP satellite signal is still mostly RHCP for low satellite elevations after reflection. However, the amplitude of the LHCP reflection coefficient is increasing with increasing satellite elevation angle, and for elevation angles over about $8^{\circ}$, the amplitude of the LHCP reflection coefficient is larger than the amplitude of the RHCP reflection coefficient (see e.g., [22,25]).

Because of the additional travel path of the reflected signals, as compared to the directly received signals, the nadir-looking antenna will in the analysis appear to be a virtual antenna located below the sea surface. The distance between the virtual antenna and the sea surface, $h_{a}$, is the same as the distance from the actual LHCP antenna to the sea surface (see Figure 2, right). This means that when there is a change in sea surface, the additional travel path of the reflected signal changes, and the LHCP antenna appears to change its vertical position. The height of the nadir-looking antenna over the sea surface $\left(h_{a}\right)$ can from the geometry presented in Figure 2 (right) be related to the vertical baseline between the two antennas as $2 h_{a}+d$, where $d$ is the vertical separation between the phase centers of the two antennas. Furthermore, the height of the nadir-looking antenna over the sea surface is directly proportional to the sea surface height.
There are several ways to analyze GNSS phase delay data in order to estimate the vertical baseline between the antennas (see e.g., $[24,30]$ ). First consider the GNSS phase observation equation expressed in meters for a single receiver and satellite, denoted with subscripts $A$ and $j$, respectively,

$$
\lambda_{i} \Phi_{A}^{j}=\varrho_{A}^{j}+c\left(\tau_{A}-\tau^{j}\right)+Z_{A}^{j}-I_{A}^{j}+\lambda_{i} N_{A}^{j}
$$

where $\lambda_{i}$ is the carrier wavelength of the GNSS signal, $\Phi_{A}^{j}$ is the observed carrier phase in units of cycles, $\varrho_{A}^{j}$ is the geometric range to the satellite, $c$ is the speed of light in vacuum, $\tau_{A}$ is the receiver clock bias, $\tau^{j}$ is the satellite clock bias, $Z_{A}^{j}$ is the delay caused by the neutral atmosphere (tropospheric delay), $I_{A}^{j}$ is the ionospheric delay, and $N_{A}^{j}$ is the phase ambiguity (including an integer number of wavelengths and unknown instrumental phase offsets from the satellite and the receiver).

Using Equation 2 for two receivers, denoted $A$ and $B$, and forming the difference result in the single difference equation for each epoch

$\lambda_{i} \Delta \Phi_{A B}^{j}=\Delta \varrho_{A B}^{j}+c \Delta \tau_{A B}+\Delta Z_{A B}^{j}-\Delta I_{A B}^{j}+\lambda_{i} \Delta N_{A B}^{j}$

where $\Delta \Phi_{A B}^{j}$ is the difference between the measured phases expressed in cycles, $\Delta \varrho_{A B}^{j}$ is the difference in geometry, $\Delta \tau_{A B}$ is the difference in receiver clock bias, $\Delta Z_{A B}^{j}$ is the difference in tropospheric delay, $\Delta I_{A B}^{j}$ is the difference in ionospheric delay, and $\Delta N_{A B}^{j}$ is the phase ambiguity difference in cycles. Note that since the difference is taken with respect to the same satellite, the differential satellite clock bias term is not present in Equation 3. Additionally, for short baselines, the tropospheric and ionospheric effects can be assumed to cancel.

For short baselines, the term for the difference in geometry (see Equation 3 ) can be expressed in a local coordinate system using the azimuth, $\alpha$, and elevation, $\epsilon$, angle for each satellite as

$$
\begin{aligned}
\Delta \varrho_{A B}^{j}= & \Delta e \sin \left(\alpha^{j}\right) \cos \left(\epsilon^{j}\right)+\Delta n \cos \left(\alpha^{j}\right) \cos \left(\epsilon^{j}\right) \\
& +\Delta v \sin \left(\epsilon^{j}\right)
\end{aligned}
$$

where $\Delta e, \Delta n$, and $\Delta v$ are the east, north, and vertical components of the baseline between the two receivers, respectively. For a known horizontal baseline (the horizontal baseline for our GNSS tide gauge is zero, see Figure 1), the east and north components can be used to adjust the left side of Equation 3.

It is also possible to form additional differences to Equation 3, e.g., double differences (between two receivers and two satellites) and triple differences (between double differences at different epochs). The double difference equation is especially advantageous for GPS observations 
for which the double differenced phase ambiguity parameters become integers (the receiver clock bias terms also cancel out). However, this is not the case for GLONASS observations, since the visible satellites have different carrier frequencies. Additionally, one extra observation is needed to form double differences as compared to single differences, and for our configuration, the number of (reflected) observations is limited. For these two reasons, we thus focused on single difference analysis.

\section{Data analysis}

The data analyses were made with in-house developed software packages in Matlab. For both the SNR analysis and the phase delay analysis, final orbits provided by the International GNSS Service (IGS) were used [31]. GPS and GLONASS data from frequency bands $\mathrm{L}_{1}$ and $\mathrm{L}_{2}$ were analyzed independently with both methods.

\subsection{SNR analysis}

Before the analysis, each SNR dataset from the zenithlooking antenna was screened to find satellite observations affected by multipath from the ocean. As a starting point, the surroundings of the GNSS tide gauge installation were inspected to determine from which directions it was possible to receive signals reflected off the sea surface. Additionally, observations from low satellite elevation angles were considered, since this is where the multipath effect is the strongest. The low satellite elevations imply that the useful observations are not reflected off the sea surface close to the antenna, but rather at some horizontal distance from the antenna. In order to determine the extent of the reflective surface, the first Fresnel zone can be used as an approximation (see e.g., $[22,25])$. The first Fresnel zone is elliptic and depends on the wavelength, the height of the antenna over the reflecting surface, and the satellite elevation angle. Examples are presented in Figure 4, depicting the first Fresnel zone for an antenna at height $4.3 \mathrm{~m}$ over the reflective surface for elevation angles of $5^{\circ}, 15^{\circ}, 30^{\circ}$, and $50^{\circ}$.
From Figure 4 it is apparent that the size of the reflective surface is decreasing for increasing satellite elevation angle. This means that for the SNR analysis, which uses observations from low satellite elevation angles, the reflective surfaces are rather large and extend from about 9 to $90 \mathrm{~m}$ from the antenna.

Taking into account that the reflective surfaces should be over the sea, it was found that satellite observations from azimuth angles of $70^{\circ}$ to $260^{\circ}$ and with elevation angles of $1^{\circ}$ to $14.5^{\circ}$ were the most affected by multipath from the ocean.

After identifying the SNR data that were strongly affected by multipath, the data were converted from $\mathrm{dB}-\mathrm{Hz}$ in logarithmic scale to watt per watt in linear scale, assuming a $1-\mathrm{Hz}$ bandwith. For each satellite arc, a second-order polynomial was fitted and removed to create $\delta$ SNR arcs. Each arc observed for longer than $10 \mathrm{~min}$ was chosen for the analysis. The reason was to ensure that several periods of the multipath oscillation were observed for each arc. These $\delta$ SNR arcs were then downsampled with a factor of 4, to speed up the analysis, and analyzed using the LSP with an oversampling factor of 40 (corresponding to a reflector height precision of about $4 \mathrm{~mm}$ ). The output from the LSP was analyzed to find the highest spectral power and the corresponding multipath frequency, i.e., the reflector height (when scaled with $\lambda_{i} / 2$ ). In order to prevent the algorithm from picking up unrealistic reflector heights, two restrictions were applied. First, the spectral power was analyzed in a window around an average frequency derived from the mean reflector height. The window was at least two times wider than the actual sea level range at the site. Second, the highest spectral power for each arc had to be at least two times larger than the mean power in the window. When these two requirements were fulfilled, the reflector heights were converted into sea level heights and time tags for each height were calculated from the mean time of the corresponding satellite arc.

In order to remove outliers, a moving average filter with a window size of five time steps (corresponding to

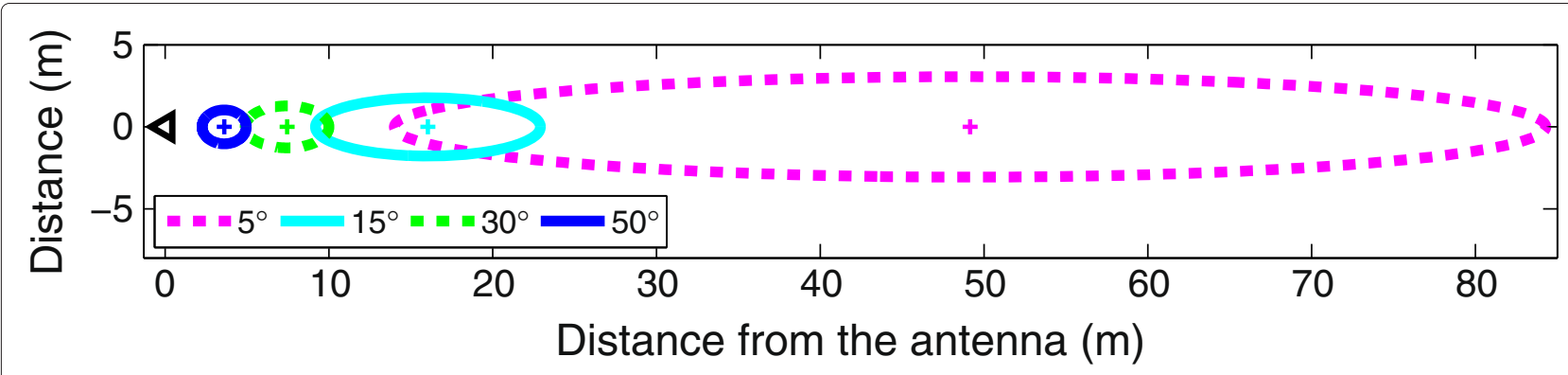

Figure 4 Extent of reflective surfaces. The reflection areas were approximated by the first Fresnel zone for an antenna at height $4.3 \mathrm{~m}$ over the reflective surface. The contours describe the reflective surfaces, for GPS observations at frequency $\mathrm{L}_{1}$ and for elevation angles $5^{\circ}$ (magenta dashed-line), $15^{\circ}$ (cyan solid line), $30^{\circ}$ (green dashed line), and $50^{\circ}$ (blue solid line). The corresponding areas are 338, 38, 10, and $4 \mathrm{~m}^{2}$, respectively. The GPS antenna is represented as a left-pointing triangle, located in the origin, and the specular point for each surface is marked with a plus sign. 
approximately 2.5 to $4 \mathrm{~h}$ ) was applied to the four sea level time series (GPS and GLONASS for frequency bands $\mathrm{L}_{1}$ and $\mathrm{L}_{2}$ ). From the difference between the original time series and the filtered time series, observations outside of $3 \sigma$ were considered as outliers and removed. For each of the SNR time series, less than $2 \%$ of the points were removed.

\subsection{Phase delay analysis}

Similar to the SNR data, the phase delay data were screened to find suitable satellite observations. In particular, the observations from the LHCP nadir-looking antenna were investigated, since these observations originate from signals reflected off the sea surface. In contrast to the SNR method, which is focused on analysis of observations from lower elevations that are highly affected by multipath, the phase delay method takes advantage of observations from higher elevations, which are less affected by multipath. The receiver connected to the zenith-looking RHCP antenna records the direct RHCP satellite signals, whereas the receiver connected to the nadir-looking LHCP antenna records the satellite signals after reflection off the sea surface (see Figure 2). After reflection, the satellite signal is dominantly LHCP for elevation angles over $8^{\circ}$.

Investigating the reflection surface, using the first Fresnel zone as in Section 4.1, for signals from higher satellite elevations, it is clear that these reflections occur close to the antenna. As an example, it is shown in Figure 4 that for an antenna at the height of $4.3 \mathrm{~m}$ above the reflector, the specular point of the reflection area is located at 7.5- and 3.6- $\mathrm{m}$ distances from the antenna for observations from elevation angles of $30^{\circ}$ and $50^{\circ}$, respectively. Taking into account that the reflective surfaces should be over the sea and that the observations from the lower elevation angles should be avoided due to the strong multipath effect, the azimuth and elevation mask were determined to be $60^{\circ}$ to $300^{\circ}$ and $15^{\circ}$ to $90^{\circ}$, respectively.

An equation system equivalent to Equation 3 was solved with a least-squares analysis for data intervals of $20 \mathrm{~min}$ at a time. Solutions were calculated for every $10 \mathrm{~min}$ using overlapping intervals and assuming that the horizontal baseline components were zero (see Equation 4). Each solution consisted of the vertical baseline component for the full interval, i.e., estimating one constant sea level component for the whole 20-min interval, the phase ambiguity differences for each satellite pair for the current interval, and the receiver clock bias differences for each epoch. For each interval, the conditions were that both receivers had continuous track of the same satellites for at least $10 \mathrm{~min}$ and that there were at least two satellites visible at each epoch. Furthermore, satellite observations had to be continuous for at least $10 \mathrm{~min}$ to be used in the processing.
The analysis resulted in time series of the vertical baseline component for the four datasets (GPS and GLONASS for frequency bands $L_{1}$ and $L_{2}$ ). In order to remove erroneous solutions and outliers, two inspections were made. First, all solutions which had a formal error in the leastsquares minimization process of larger than $4 \mathrm{~cm}$ were removed. Second, a moving average filter with a window size of 21 time steps (corresponding to approximately $3.5 \mathrm{~h}$ ) was applied to each time series. From the difference between the original time series and the filtered time series, observations outside of $3 \sigma$ were considered as outliers and removed. For each of the phase delay time series, less than $12 \%$ of the points were removed.

The vertical baselines (the solutions) for each dataset were converted into sea level heights relative to the nadirlooking antenna. Note that the vertical distance between the phase centers of the two antennas was not accounted for, causing a bias.

\section{Results}

In the SNR analysis, only the geometrical component of the signal was considered and not any effects originating from the surface composition and antenna response, which can change the phase of the reflected signal (see [32]). Similarly, for the phase delay analysis, signal phase change from the reflection, e.g., carrier phase wind-up [33], and antenna phase center variations were not taken into account. Therefore, the results derived from the two analysis methods were not compared in an absolute sense. Instead, relative GNSS sea level time series were compared to the independent sea level observations from the co-located tide gauge. The tide gauge consisted of three pressure sensors (see Section 2). In a comparison with a pneumatic bubbler gauge, installed after this campaign, it was found that all three pressure sensors showed systematic errors (see [34]). However, one of the sensors showed only minor errors, and therefore, that sensor was used as the reference tide gauge in this study.

Since the GNSS-derived sea level measurements are relative to the phase center of the antenna and the tide gauge sea level observations were relative to the sensor installation, a mean was removed from each time series before comparison. This means that only the relative variations in sea level were compared and not the absolute sea level values.

In order to illustrate the daily variations in sea level for each method, subsets of about 2 days (from the original 30 days) of sea level from the SNR analysis and the phase delay analysis are displayed in Figures 5 and 6, respectively. For the ease of viewing, the time series are offset by $40 \mathrm{~cm}$ and consist, from top to bottom, of data from GPS $\mathrm{L}_{1}$, GLONASS $\mathrm{L}_{1}$, GPS $\mathrm{L}_{2}$, and GLONASS $\mathrm{L}_{2}$. In addition, the tide gauge time series is presented together with each GNSS-derived time series. 


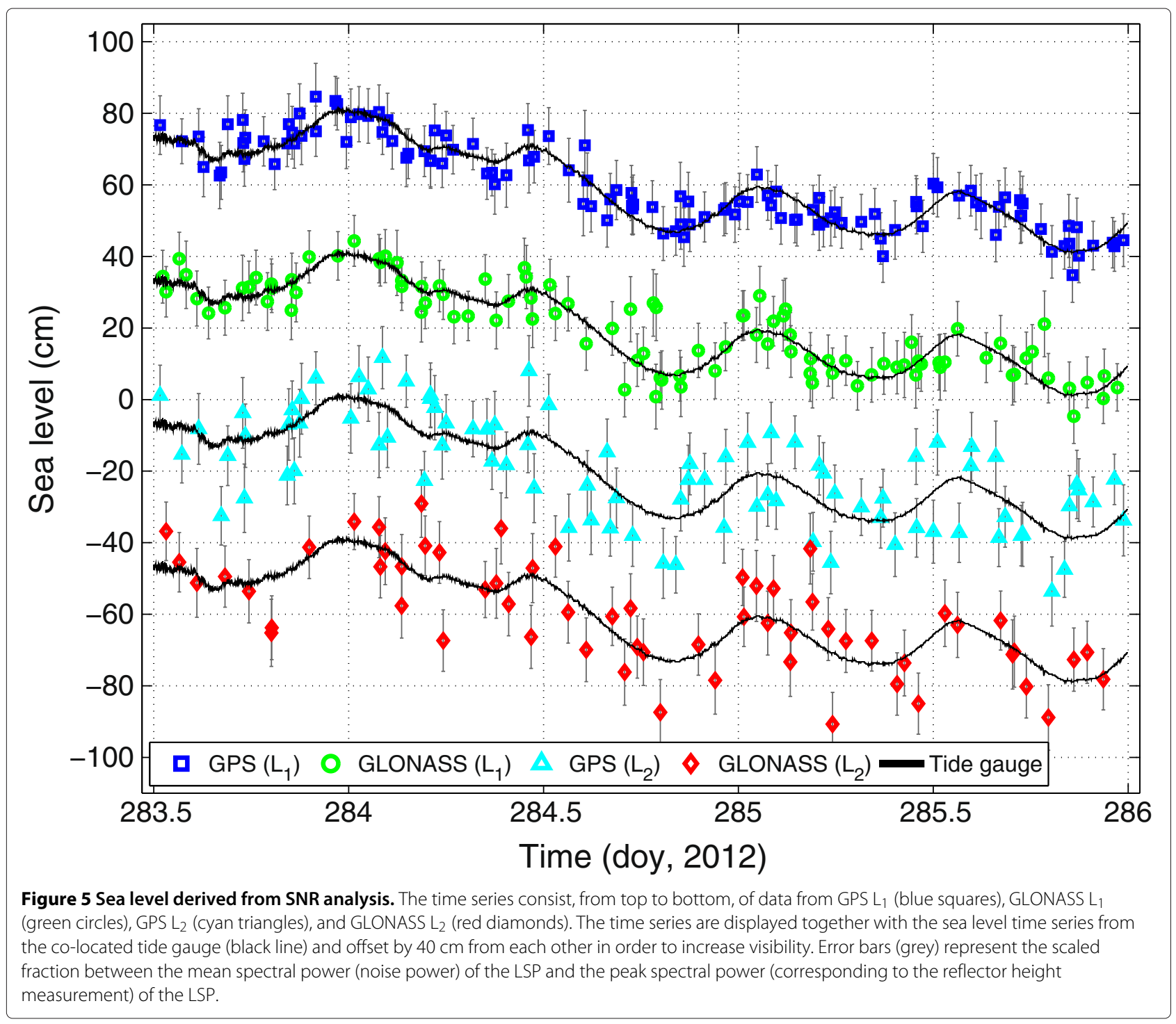

In Figure 5, the SNR sea level derived with $\mathrm{L}_{1}$ data from both GPS and GLONASS (top two time series) shows good agreement with the tide gauge sea level. The sea level solutions derived from $\mathrm{L}_{2}$ data (bottom two time series) are more noisy than those derived from $\mathrm{L}_{1}$ data, but they do appear to follow the tide gauge sea level rather well. Since each sea level value is derived from the corresponding multipath oscillation frequency, it is not trivial to assess the accuracy of each estimation. As an approximation of the formal error, the fraction between the mean spectral power (noise power) of the LSP and the peak spectral power (corresponding to the reflector height measurement) of the LSP is taken and multiplied with a factor of 0.2 (see the grey error bars in Figure 5).

The phase delay sea level results in Figure 6 resemble the tide gauge sea level well for GPS and GLONASS at both frequencies. The error bars consist of the standard deviation (the formal error in the least-squares minimization process) multiplied by a factor of 10 .

Comparing the SNR and the phase delay results from Figures 5 and 6, which are shown in the same scale, it is clear that the phase delay sea level results are in general consistently in better agreement with the tide gauge sea level than the SNR sea level results are. In order to evaluate the GNSS-derived sea level in a more qualitative way, the Van de Casteele test was used (see e.g., [35]). The Van de Casteele test is a simple diagram based on simultaneous sea level height measurements from a reference tide gauge (here the pressure tide gauge) and from a tide gauge to be tested (here the GNSS tide gauge). Sea level height is presented along the $y$-axis of the diagram, and the tide gauge error, i.e., the difference in sea level height between the two sea level records, is presented 


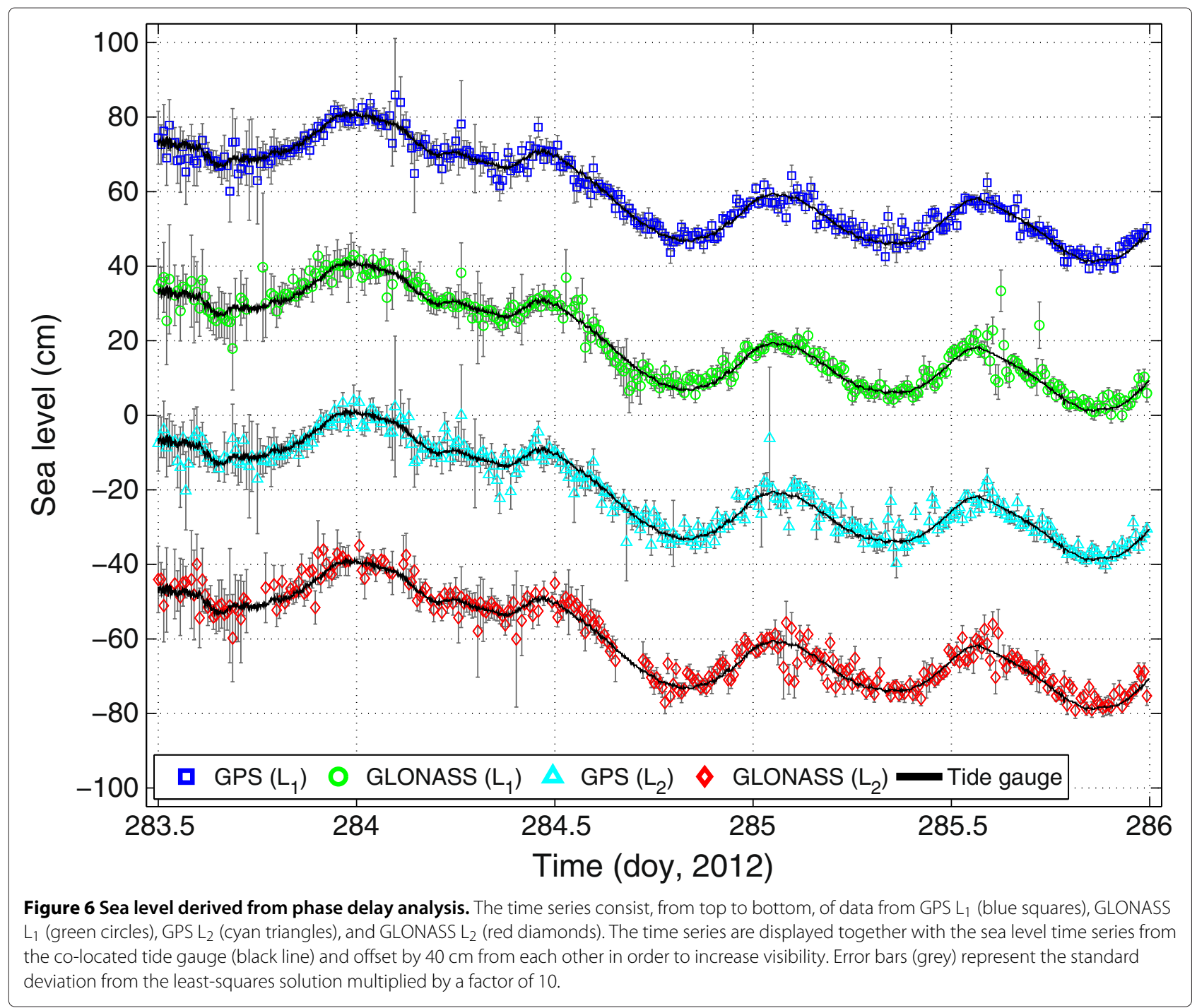

along the $x$-axis of the diagram. From the shape of the Van de Casteele diagram, it is possible to easily understand whether systematic errors exist.

In order to construct Van de Casteele diagrams, the tide gauge sea level record was resampled to the nearest epochs of each GNSS-derived sea level record. As an example of the Van de Casteele results, Figure 7 depicts the diagram for GPS $\mathrm{L}_{1}$ (left) and for GLONASS $\mathrm{L}_{1}$ (right). The results are shown for sea level heights from both the SNR analysis (blue) and the phase delay analysis (green).

From Figure 7, it is again apparent that the phase delay sea level better resembles the tide gauge sea level, i.e., the tide gauge error is smaller, than the SNR sea level. The SNR sea level appears to be a bit more noisy than the phase delay sea level. However, there seems to be a few large outliers in the phase delay results. The shapes of the four Van de Casteele diagrams, centered around zero tide gauge error, indicate that there are no systematic errors for the GNSS-derived sea level. The corresponding four Van de Casteele diagrams for the $\mathrm{L}_{2}$ frequency band gave similar results and did not show any systematic errors for the GNSS-derived sea level either. In addition, no major differences were found between GPS and GLONASS results for the phase delay analysis or for the SNR analysis.

For a quantitative comparison between the GNSSderived sea level time series and the reference tide gauge record, correlation coefficients, root-mean-square (RMS) differences, and pairwise mean (absolute) differences were calculated. The results are presented, together with the number of data points that were available for each comparison, in Table 1.

First of all, in Table 1, the number of points for each GNSS solution used in the comparison was similar, except for GLONASS $\mathrm{L}_{2}$ which had a lower number of solutions. For the SNR method, the number of solutions depends on the number of satellites that rise or set over the ocean 


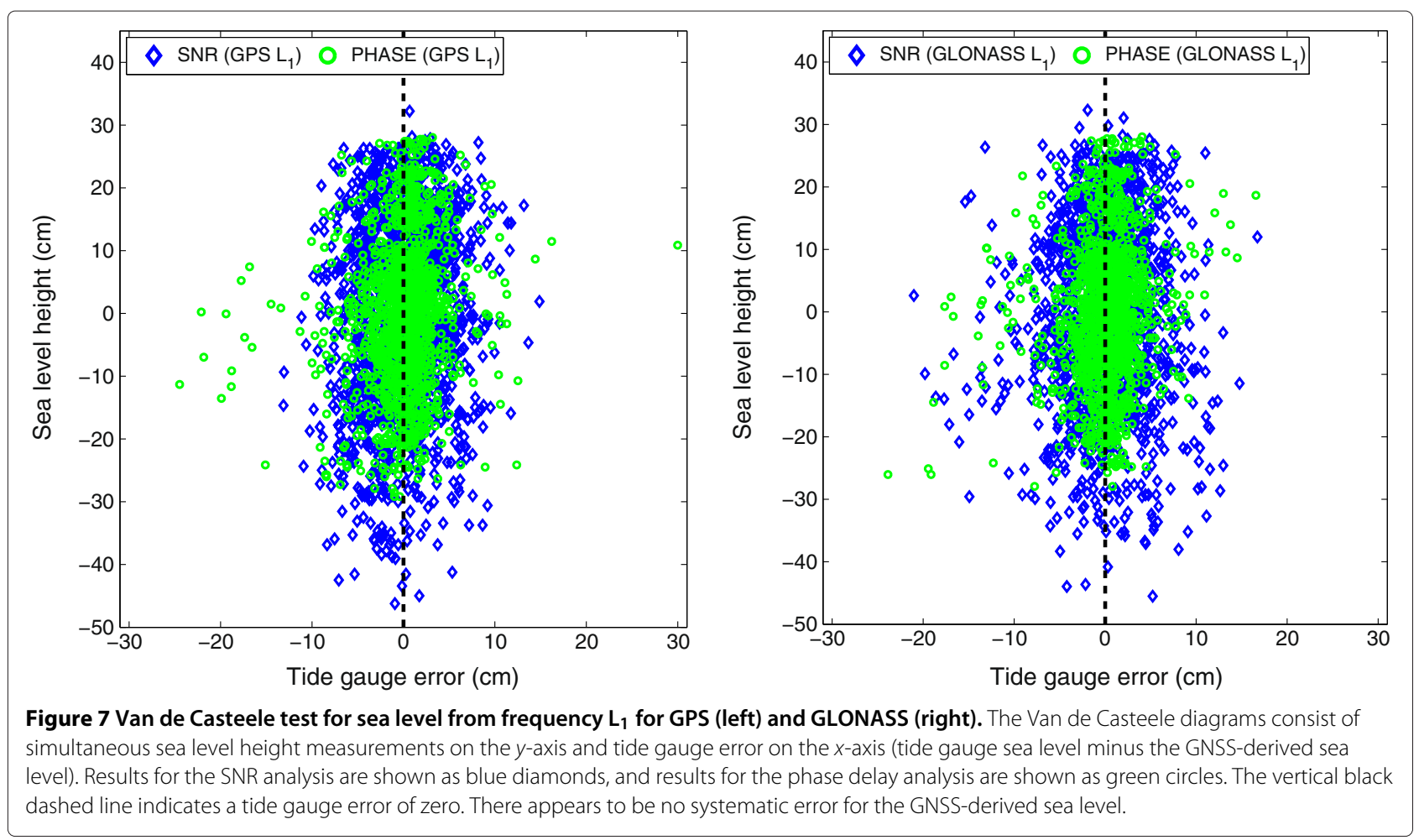

everyday. Since GLONASS has about 30\% fewer orbiting satellites than GPS, it is also reasonable that there are correspondingly less GLONASS than GPS SNR solutions. The number of SNR $\mathrm{L}_{1} / \mathrm{L}_{2}$ solutions per day was on average 49/40 and 40/28 for GPS and GLONASS, respectively, i.e., about $20 \%$ to $40 \%$ less for GLONASS compared to GPS. In addition, the signals from frequency band $L_{2}$ are weaker in signal strength than the signals from frequency band $L_{1}$. This means that the SNR is lower and thus the

Table 1 Comparison between the GNSS-derived sea level time series and the reference tide gauge record

\begin{tabular}{ccccccc}
\hline & & \multicolumn{2}{c}{ GPS } & & \multicolumn{2}{c}{ GLONASS } \\
\cline { 3 - 4 } \cline { 7 - 7 } & & $\mathbf{L}_{\mathbf{1}}$ & $\mathbf{L}_{\mathbf{2}}$ & & $\mathbf{L}_{\mathbf{1}}$ & $\mathbf{L}_{\mathbf{2}}$ \\
\hline \multirow{3}{*}{ SNR } & Points (nr) & 1,516 & 1,229 & & 1,254 & 882 \\
& Corr. coeff. & 0.97 & 0.86 & & 0.96 & 0.87 \\
& RMS & 4.0 & 9.0 & & 4.7 & 8.9 \\
& Mean diff. & 3.2 & 7.5 & & 3.6 & 7.0 \\
& & & & & \\
Phase & Points (nr) & 1,534 & 1,495 & & 1,408 & 1,286 \\
delay & Corr. coeff. & 0.95 & 0.95 & & 0.96 & 0.96 \\
& RMS & 3.5 & 3.5 & & 3.3 & 3.2 \\
& Mean diff. & 2.3 & 2.4 & & 2.2 & 2.3 \\
\hline
\end{tabular}

The table shows the correlation coefficients, RMS differences, mean (absolute) differences, and the number of data points available for the comparison, for sea level from SNR analysis and the phase delay analysis of GPS and GLONASS $L_{1}$ and $L_{2}$ data compared with tide gauge records. All RMS values and differences are given in centimeters. reflector height determination is more difficult using the $\mathrm{L}_{2}$ signals.

For the phase delay method, the number of solutions can be up to the sampling rate of the receiver. In this study, solutions were produced every $10 \mathrm{~min}$ (144 solutions per day). However, as reported before by [22], the geodetic receiver connected to the nadir-looking antenna has problems with keeping track of the satellite signals when the sea surface is rough, which usually corresponds to wind speeds of about 7 to $9 \mathrm{~m} / \mathrm{s}$. This means that for these conditions, there are no phase delay solutions.

Both analysis methods show high correlation with the independent tide gauge record, resulting in correlation coefficients of 0.95 to 0.97 . The exception is the SNR technique for frequency, with correlation coefficients of 0.86 to 0.87 (see Table 1). The phase delay analysis performs similarly for both frequencies and for both systems with RMS differences of $3.5 \mathrm{~cm}$ for GPS and 3.2 to $3.3 \mathrm{~cm}$ for GLONASS. As previously mentioned, the results from phase delay analyses show an overall better agreement with the tide gauge sea level than the results from SNR analyses. The SNR analysis achieves an RMS difference of 4.0 to $4.7 \mathrm{~cm}$ for frequency $\mathrm{L}_{1}$, whereas the RMS difference is around $9.0 \mathrm{~cm}$ for frequency $\mathrm{L}_{2}$.

\section{Discussion}

The two GNSS, GPS and GLONASS, have a similar performance for both analysis methods. However, there are some built-in differences for the two systems: the number 
of orbiting satellites, which is higher for GPS, and the multiple access technique used. The former impacts, for the SNR analysis, the number of possible sea level solutions per day and, for the phase delay analysis, the robustness of each sea level solution (number of observations in the least-squares solution). The two multiple access techniques used for GPS and GLONASS are code division multiple access (CDMA) and frequency division multiple access (FDMA), respectively. This means that all GPS satellites use the same $L_{1}$ and $L_{2}$ carrier frequencies, whereas GLONASS satellites have slightly different carrier frequencies, separated by multiples of 562.5 and $437.5 \mathrm{kHz}$ for $L_{1}$ and $L_{2}$, respectively. Furthermore, the GPS carrier frequencies are lower than the GLONASS carrier frequencies.

The different frequencies imply that the size of the reflection area, or footprint, is different for different satellites. However, the difference is very small, e.g., approximating the reflection surface with the first Fresnel zone shows that the maximum difference in footprint, at the same frequency band, for an installation of height $4.3 \mathrm{~m}$ over the sea surface, and at elevation angles of $5^{\circ}, 10^{\circ}$, and $15^{\circ}$, is $7.3,1.8$, and $0.8 \mathrm{~m}^{2}$, respectively. This is much smaller than the actual size of the footprint and the difference decrease with increasing elevation angle.

An additional difference regarding satellite footprints is the repetition frequency of each footprint. For the GPS, the satellite constellation repeats approximately every 12 sidereal hours, which means that the footprint repeats with the same period [24] and each satellite will illuminate the same area every orbit. However, for GLONASS, the satellite constellation repeats approximately every 8 sidereal days. Thus, the combined use of GPS and GLONASS gives both a better temporal resolution (more sea level observations per time unit) and spatial resolution (better sea surface coverage per time unit) than each system alone.

It was previously mentioned that the number of solutions for the phase delay analysis decreases when the sea surface gets rougher (due to loss of lock on the satellite signals for the receiver connected to the nadir-looking antenna). Since the data used for the SNR analysis are recorded by the receiver connected to the zenith-looking antenna, the problem with loss of lock does not occur with this method. Nonetheless, it is of importance to investigate if there is any effect on the solutions of the SNR analysis from rough sea surfaces. Instead of using measurements of sea surface roughness, wind speed measurements from OSO (approximately $200 \mathrm{~m}$ away from the GNSS tide gauge installation) were used to indicate sea surface roughness, similar to [22]. The tide gauge error, i.e., the difference between the reference pressure tide gauge record and the GNSS-derived sea level, was then compared to the wind speed for identical epochs (wind speed measurements are available every $10 \mathrm{~min}$ ). The tide gauge error for GPS $\mathrm{L}_{1}$ compared to wind speed, both for SNR analysis and phase delay analysis, is presented in Figure 8.

In Figure 8, the tide gauge errors of the SNR solution are distributed around zero for wind speeds between 0.5 and $17.5 \mathrm{~m} / \mathrm{s}$, which were the lowest and highest wind speeds observed during the campaign, respectively. It does not appear that there are significantly less SNR-derived results at higher wind speeds than at lower wind speeds, and there is no visible correlation between the tide gauge error and increasing wind speed. However, the number of phase delay solutions decreases around wind speeds of $6 \mathrm{~m} / \mathrm{s}$, and no phase delay results are available for wind speeds of over $11 \mathrm{~m} / \mathrm{s}$, which is consistent with [22]. The corresponding graphs for the GNSS sea level results derived from frequency $L_{2}$ observations show similar results.

The reason that the SNR analysis results do not appear to be affected by an increase in sea surface roughness, or wind speed, is most probably due to the receiver, which is locking on to the direct satellite signal (or actually the composite signal comprised by the direct and the multipath signals). The amplitude of the SNR oscillations depends on both the direct and reflected signal power (see e.g., [28]) and will therefore decrease with a decreasing signal power from the reflected signal, i.e.,

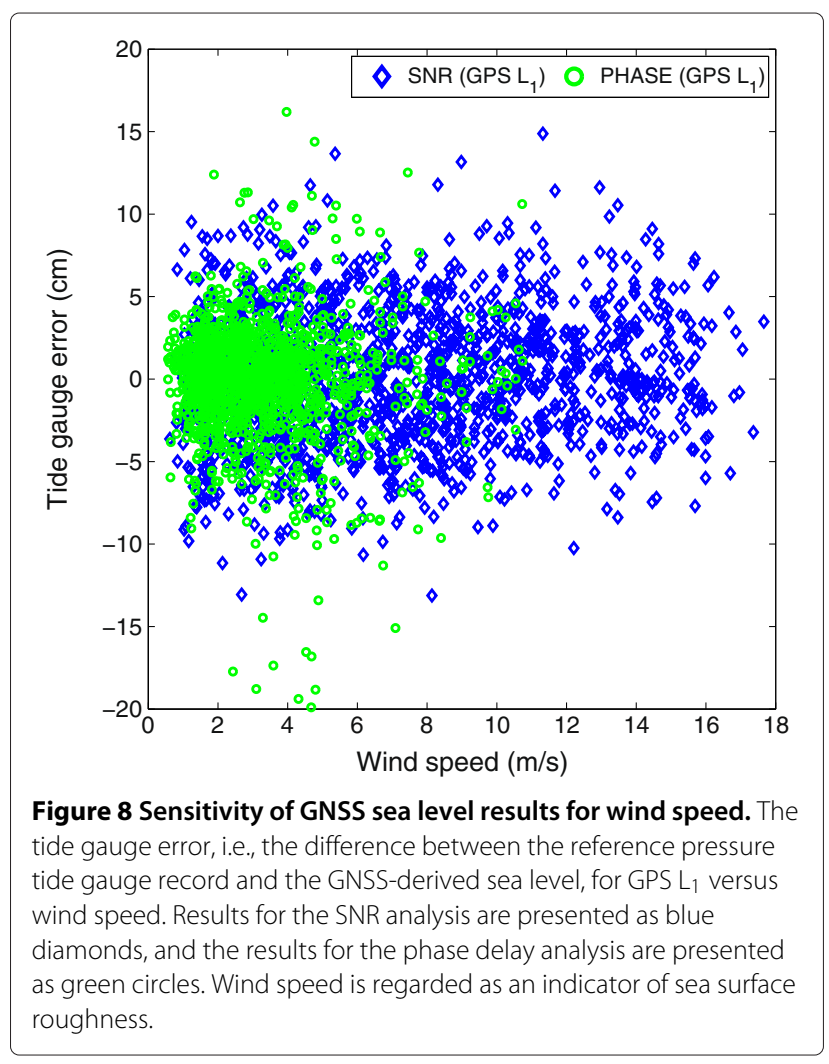


with increasing sea surface roughness. However, even if the reflected signal is weaker, it will still interfere with the direct signal, creating oscillations in the SNR data. In addition, the sea surface roughness effect on the reflected signal is not linearly dependent on the wind speed.

\section{Conclusions}

The aim of this study was to evaluate and compare sea level solutions from two analysis methods (SNR analysis and phase delay analysis), two GNSS (GPS and GLONASS), and two carrier frequency bands $\left(\mathrm{L}_{1}\right.$ and $\left.\mathrm{L}_{2}\right)$. The SNR analysis uses multipath signals observed with an upward-looking antenna, and the phase delay analysis uses the phase delay for both an upward-and a downwardlooking antenna. This study has been made possible with the GNSS tide gauge installation at OSO, which is equipped with both a zenith-looking RHCP antenna and a nadir-looking LHCP antenna. The two antennas are both connected to a geodetic GNSS receiver each. Both GPS and GLONASS $\mathrm{L}_{1}$ and $\mathrm{L}_{2}$ signals were recorded during 1 month and analyzed with both analysis methods, and the results were compared in a relative sense to independent measurements of sea level from a co-located pressure tide gauge.

The GNSS-derived sea level shows a high correlation with the tide gauge sea level for both analysis methods. Correlation coefficients for the phase delay analysis and for the SNR analysis using frequency $\mathrm{L}_{1}$ are 0.95 to 0.97 , whereas the correlation coefficients for the SNR analysis using frequency $\mathrm{L}_{2}$ are 0.86 to 0.87 .

Comparing the results from the SNR analysis and the phase delay analysis for frequency $L_{1}$, it is clear that the sea level from phase delay analysis shows a better agreement with the independent tide gauge sea level than the sea level from SNR analysis. Expressed as RMS differences, the phase delay analysis achieves values of $3.5 \mathrm{~cm}$ (GPS) and $3.3 \mathrm{~cm}$ (GLONASS), whereas the SNR analysis achieves higher values of $4.0 \mathrm{~cm}$ (GPS) and $4.7 \mathrm{~cm}$ (GLONASS). The lower RMS difference for the phase delay analysis as compared to the SNR analysis is consistent with [14], where the GPS $\mathrm{L}_{1}$ results were compared to a synthetic tide gauge. In this study, we additionally show that the phase delay analysis results for frequency $\mathrm{L}_{2}$ are on the same level of precision as those of frequency $\mathrm{L}_{1}$ with RMS differences of $3.5 \mathrm{~cm}$ (GPS) and $3.2 \mathrm{~cm}$ (GLONASS). However, the results derived from the SNR analysis at frequency $\mathrm{L}_{2}$ give larger RMS differences than for $\mathrm{L}_{1}$ with values of $9.0 \mathrm{~cm}$ (GPS) and $8.9 \mathrm{~cm}$ (GLONASS).

The two GNSS show a similar performance when compared to the tide gauge record. From the results, our conclusions are that, for the phase delay analysis, it is possible to use both frequency bands and, for the SNR analysis, frequency band $\mathrm{L}_{2}$ should be avoided if other signals are available. Note that standard geodetic receivers using code-based tracking, i.e., tracking the un-encrypted $\mathrm{C} / \mathrm{A}$-code on $\mathrm{L}_{1}$ and using the manufacturers' proprietary tracking method for $\mathrm{L}_{2}$, were used. Signals with the new $\mathrm{C} / \mathrm{A}$-code on $\mathrm{L}_{2}$, the so-called $\mathrm{L}_{2 C}$, have proven useful for SNR analysis in previous studies (see e.g., [14,27]), but these signals were not used in this study.

It has previously been shown in [22] that the phase delay method for GPS frequency $L_{1}$ has difficulties during rough sea conditions, as indicated by a decreasing number of solutions for wind speeds between 7 and $9 \mathrm{~m} / \mathrm{s}$. In this study, this result is confirmed by investigating the difference between the GNSS $\mathrm{L}_{1} / \mathrm{L}_{2}$ phase delay sea level and the tide gauge sea level for increasing wind speeds. In addition, the same investigation was made with the SNR method for GNSS $\mathrm{L}_{1} / \mathrm{L}_{2}$. The latter results showed that no visible effects were found for wind speeds up to $17.5 \mathrm{~m} / \mathrm{s}$ (which was the maximum wind speed observed during the campaign).

For the future, we anticipate multi-GNSS solutions, i.e., combining several GNSS. For example, using both GPS and GLONASS signals together will increase the number of observations in a combined phase delay analysis, providing more accurate sea level estimates. The combination of GPS and GLONASS for the SNR analysis will increase the temporal resolution of the corresponding sea level results. Additionally, an improved handling of antenna phase center variations and signal reflection effects will allow time series of absolute sea level.

The next level of combination will be to use multiGNSS, multi-frequency, phase delay, and SNR analysis in a filter approach, in order to benefit from the individual advantages. Doing so, we expect that it will be possible to derive continuous and accurate absolute GNSS sea level time series in a wide range of wind speeds.

\section{Competing interests}

The authors declare that they have no competing interests.

\section{Acknowledgements}

The authors would like to thank Mattias Bermell Rudfelt, Daniel Hermansson, Annika Johansson, and Otto Torgnyson for their Bachelor thesis on analysis of SNR observations from GPS and GLONASS.

Received: 2 December 2013 Accepted: 21 March 2014

Published: 11 April 2014

\section{References}

1. M Martin-Neira, A Passive Reflectometry and Interferometry System (PARIS): application to ocean altimetry. ESA J. 17, 331-355 (1993)

2. M Caparrini, A Egido, F Soulat, O Germain, E Farres, S Dunne, G Ruffini, Oceanpal ${ }^{\circledR}$ : monitoring sea state with a GNSS-R coastal instrument. Paper presented at the International Geoscience and Remote Sensing Symposium, IEEE, Barcelona, Spain, 23-28 July 2007, doi:10.1109/IGARSS.2007.4424004

3. ST Lowe, C Zuffada, Y Chao, P Kroger, LE Young, 5-cm-Precision aircraft ocean altimetry using GPS reflections. Geophys. Res. Lett. 29(10), 1375 (2002). doi:10.1029/2002GL014759 
4. S Gleason, S Hodgart, Y Sun, C Gommenginger, S Mackin, M Adjrad, M Unwin, Detection and processing of bistatically reflected GPS signals from low Earth orbit for the purpose of ocean remote sensing.Trans. Geosci. Remote Sensing. 43(6), 1229-1241 (2005). doi:10.1109/TGRS.2005. 8456432

5. NL Bindoff, J Willebrand, V Artale, A Cazenave, J Gregory, S Gulev, K Hanawa, C Le Qur, S Levitus, Y Nojiri, CK Shum, LD Talley, A Unnikrishnan, Observations: Oceanic Climate Change and Sea Level, in Climate Change 2007: The Physical Science Basis, Contribution of Working Group I to the Fourth Assessment Report of the Intergovernmental Panel on Climate Change. (S Solomon, D Qin, M Manning, Z Chen, M Marquis, KB Averyt, M Tignor, HL Miller, eds.) (Cambridge University Press, Cambridge, United Kingdom and New York, NY, USA, 2007)

6. M Lidberg, JM Johansson, H-G Scherneck, GA Milne, Recent results based on continuous GPS observations of the GIA process in Fennoscandia from BIFROST. J. Geod. 50(1), 8-18 (2010). doi:10.1016/j.jog.2009.11.010

7. G Wöppelmann, C Letetrel, A Santamaria, M-N Bouin, X Collilieux, Z Altamimi, SDP Williams, B Martin Miguez, Rates of sea-level change over the past century in a geocentric reference frame. Geophys. Re. Lett. 36, L12607 (2009). doi:10.1029/2009GL038720

8. T Schöne, N Schön, D Thaller, IGS Tide Gauge benchmark monitoring pilot project (TIGA): scientific benefits. J. Geod. 83, 249-261 (2009) doi:10.1007/s00190-008-0269-y

9. Y Georgiadou, A Kleusberg, On carrier signal multipath effects in relative GPS positioning. Manusc. Geod. 13, 172-179 (1988)

10. P Elósegui, JL Davis, RTK Jaldehag, JM Johansson, AE Niell, II Shapiro, Geodesy using the Global Positioning System: the effects of signal scattering on estimates of site position. J. Geophys. Res. 100. B6, 2156-2202 (1995). doi:10.1029/95JB00868

11. KD Anderson, A GPS tide gauge. GPS World Showcase. 6, 44-44 (1995)

12. KD Anderson, Remote Sensing: A Valuable Source of Information, Paper presented at the AGARD SPP Symposium. Toulouse, France, 22-25 April 1996

13. KD Anderson, Determination of water level and tides using interferometric observations of GPS Signals. J. Atmos. Oceanic Technol. 17(8), 1118-1127 (2000). doi:10.1175/1520-0426(2000)017<1118:DOWLAT>2.0.CO;2

14. KM Larson, JS Löfgren, R Haas, Coastal sea level measurements using a single geodetic GPS receiver. Adv. Space Res. 51 (8), 1301-1310 (2013). doi:10.1016/j.asr.2012.04.017

15. KM Larson, RD Ray, FG Nievinski, JT Freymueller, The accidental tide gauge: a GPS reflection case study from Kachemak Bay Alaska. IEEE Geosci. Remote Sens. Lett. 10(5), 1200-1204 (2013). doi:10.1109/LGRS. 2012.2236075

16. JS Löfgren, R Haas, H-G Scherneck, Sea level time series and ocean tide analysis from multipath signals at five GPS sites in different parts of the world. J. Geodyn., in press (2014). doi:10.1016/j.jog.2014.02.012

17. N Rodriguez-Alvarez, X Bosch-Lluis, A Camps, I Ramos-Perez, E Valencia, H Park, M Vall-Ilossera, Water level monitoring using the interference pattern GNSS-R technique, in IEEE Geoscience and Remote Sensing Symposium (IGARSS). Vancouver, 24-29 July 2011, pp. 2334-2337. doi:10.1109/IGARSS.2011.6049677

18. S Dunne, F Soulat, M Caparrini, O Germain, E Farres, X Barroso, G Ruffini, Oceanpal ${ }^{\oplus}$, a GPS-reflection coastal instrument to monitor tide and sea-state. Paper presented at Oceans 2005 - Europe 2. Brest, France, 20-23 June 2005. doi:10.1109/OCEANSE.2005.1513257

19. M Belmonte Rivas, M Martin-Neira, Coherent GPS reflections from the sea surface. IEEE Geosci. Remote Sens. Lett. 3, 28-31 (2006). doi:10.1109/LGRS. 2005.855617

20. M Martin-Neira, P Colmenarejo, G Ruffini, C Serra, Altimetry precision of 1 $\mathrm{cm}$ over a pond using the wide-lane carrier phase of GPS reflected signals. Can. J. Rem. Sens. 28(3), 394-403 (2002). doi:10.5589/m02-039

21. JS Löfgren, R Haas, JM Johansson, Monitoring coastal sea level using reflected GNSS signals. J. Adv. Space Res. 47(2), 213-220 (2011). doi:10.1016/j.asr.2010.08.015

22. JS Löfgren, R Haas, H-G Scherneck, MS Bos, Three months of local sea level derived from reflected GNSS signals. Radio Sci. 46, RSOC05 (2011). doi:10.1029/2011RS004693

23. Intergovernmental Oceanographic Commission (IOC) of UNESCO, Manual on Sea-level Measurements and Interpretation, Volume IV: An upyear to 2006, IOC Manuals and Guides No.14, vol. IV. (JCOMM Technical Report No.31; WMO/TD. No.1339, 78 pp, Paris, 2006)
24. B Hofmann-Wellenhof, H Lichtenegger, J Collins, GPS Theory and Practice, 5th edn. (Springer, Wien, New York, 2001), ISBN 3-211-83534-2

25. BM Hannah, Modelling and simulation of GPS multipath propagation. Dissertation, Queensland University of Technology, 2001

26. KM Larson, EE Small, ED Gutmann, AL Bilich, JJ Braun, VU Zavorotny, Use of GPS receivers as a soil moisture network for water cycle studies. Geophys. Res. Lett. 35, L24405 (2008). doi:10.1029/2008GL036013

27. KM Larson, ED Gutmann, VU Zavorotny, JJ Braun, MW Williams, FG Nievinski, Can we measure snow depth with GPS receivers? Geophys. Res. Lett. 36, L17502 (2009). doi:10.1029/2009GL039430

28. A Bilich, KM Larson, Mapping the GPS multipath environment using the signal-to-noise ratio (SNR). Radio Sci. 42, RS6003 (2007). doi:10.1029/2007RS003652

29. CJ Benton, CN Mitchell, Isolating the multipath component in GNSS signal-to-noise data and locating reflecting objects. Radio Sci. 46, RS6002 (2011). doi:10.1029/2011RS004767

30. G Blewitt, Basics of the GPS technique: observation equations, in Geodetic Applications of GPS. ed. by B Johnson, (Nordic Geodetic Commission Sweden, 1997). ISSN 0280-5731, 10-54

31. JM Dow, RE Neilan, C Rizos, The International GNSS Service in a changing landscape of Global Navigation Satellite Systems. J. Geod. 83, 191-198 (2009). doi:10.1007/s00190-008-0300-3

32. FG Nievinski, Forward and inverse modeling of GPS multipath for snow monitoring. Dissertation, Department of Aerospace Engineering Sciences, University of Colorado, 2013

33. G Beyerle, Carrier phase wind-up in GPS reflectometry. GPS Solut. 13, 191-198 (2009). doi:10.1007/s10291-008-0112-1

34. JS Löfgren, Local sea level observations using reflected GNSS signals, Dissertation, Department of Earth and Space Sciences. Chalmers University of Technology, 2014

35. BM Miguez, L Testut, G Wöppelmann, The Van de Casteele test revisited an efficient approach to tide gauge error characterization. J. Atmos. Oceanic Technol. 25, 1238-1244 (2008). doi:10.1175/2007JTECHO554.1

doi:10.1186/1687-6180-2014-50

Cite this article as: Löfgren and Haas: Sea level measurements using multifrequency GPS and GLONASS observations. EURASIP Journal on Advances in Signal Processing 2014 2014:50.

\section{Submit your manuscript to a SpringerOpen ${ }^{\circ}$ journal and benefit from:}

- Convenient online submission

- Rigorous peer review

- Immediate publication on acceptance

- Open access: articles freely available online

- High visibility within the field

- Retaining the copyright to your article

Submit your next manuscript at $\boldsymbol{\nabla}$ springeropen.com 